\title{
The first case of cutaneous infection with Mycobacterium parascrofulaceum
}

This article was published in the following Dove Press journal:

Therapeutics and Clinical Risk Management

16 August 2012

Number of times this article has been viewed

\author{
Wenkai Zong* \\ Xiaodong Zhang* \\ Hongsheng Wang \\ Xiu Lian Xu \\ Qiuling Wang \\ Weiwei Tian \\ Ya Li Jin \\ Qinxue Wu \\ Meiyu Tang
}

Institute of Dermatology, Chinese Academy of Medical Sciences and Peking Union Medical College,

National Center for STD and Leprosy

Control, Chinese Center for Disease

Control and Prevention, Nanjing,

People's Republic of China

*These authors contributed equally to this work

\begin{abstract}
The authors present the first, to the best of their knowledge, reported case of cutaneous infection caused by Mycobacterium parascrofulaceum. A 42-year-old woman presented with asymptomatic reddish papules, nodules, plaques, and patches on the right side of her face and on her forehead that had persisted for 5 years, with the lesions gradually increasing in size over that time. No previous intervening medical treatment had been applied. No history or evidence of immunosuppression was found. A skin biopsy was performed for routine histological examination. Samples of lesioned skin were inoculated on Löwenstein-Jensen medium to determine the presence of acid-fast bacilli. Ziehl-Neelsen staining was used to confirm the presence of the organism. In vitro drug susceptibility testing was conducted using the microtiter plate method. Mycobacterium was identified by polymerase chain reaction-restriction fragment length polymorphism analysis and sequencing of the hsp65 and $16 S$ rDNA genes. Cultures for aerobic and anaerobic bacteria, as well as fungus, were also conducted. Routine histopathology revealed granulomatous changes without caseation. Ziehl-Neelsen staining showed that the organisms in both the lesions and the cultures were acid-fast bacilli. The cultured colonies were grown in Löwenstein-Jensen medium and incubated at two different temperatures $\left(32^{\circ} \mathrm{C}\right.$ and $37^{\circ} \mathrm{C}$ ) for $2-3$ weeks, developing pigmentation both in the dark and in the light. In vitro drug susceptibility tests showed that the organism was sensitive to clarithromycin and moxifloxacin. Polymerase chain reaction-restriction fragment length polymorphism analysis and sequencing of the hsp 65 and $16 S r D N A$ genes confirmed that the isolated organisms were M. parascrofulaceum. Fungal and other standard bacterial cultures were negative. In conclusion, identification and diagnosis of nontuberculous mycobacteria should be performed promptly to obtain better prognoses. Empirical treatments may be feasible, and drug susceptibility tests are important.

Keywords: nontuberculous mycobacteria, skin infection, PCR-RFLP, laboratory diagnosis, therapy
\end{abstract}

\section{Introduction}

Nontuberculous mycobacteria (NTM) are acid-fast mycobacteria, except for Mycobacterium tuberculosis and Mycobacterium leprae, and include over 100 species; the number of these species has significantly increased in recent years. ${ }^{1}$ NTM are environmental pathogens distributed via water, wet soil, house dust, dairy products, cold-blooded animals, vegetation, human feces, and so on. ${ }^{2}$ Many NTM are avirulent and cause no disease. Several NTM can cause opportunistic infections in immunosuppressed patients and, occasionally, in normal hosts. Given the increasing number of both acquired immune deficiency syndrome (AIDS) patients and cases of immunosuppressive drug abuse, the number of NTM infections has risen quickly in recent decades. 
Treatment is difficult because NTM infections generally respond poorly to antituberculous therapy. As treatment and prognosis both depend on the responsible organism being obtained from tissue specimens, both recognition and identification of NTM species are becoming increasingly important. Molecular methods, such as polymerase chain reaction (PCR)-based techniques and genetic sequencing, have recently been developed and are important tools for mycobacterial differentiation. PCR-restriction fragment length polymorphism (PCR-RFLP) presents fewer methodological variations or misinterpretations than conventional procedures and thus has the potential to provide more accurate identification. ${ }^{3}$

Mycobacterium parascrofulaceum, a new species of NTM belonging to group II, was first described by Turenne et $\mathrm{al}^{4}$ in 2004. Similar to the majority of NTM, M. parascrofulaceum is an opportunistic species found in the environment. However, infection from this species is rare and thus infrequently reported; as such, all relevant reports to date are limited to humans. This strain usually causes respiratory diseases, especially in elderly immunosuppressed patients with predisposing factors. ${ }^{4-8}$ The authors present the first, to the best of their knowledge, reported case of cutaneous infection caused by M. parascrofulaceum in a patient without immunosuppression.

\section{Case report}

A 42-year-old woman presented with asymptomatic reddish papules, nodules, plaques, and patches on the right side of her face and on her forehead that had persisted for 5 years. These lesions had initially appeared as reddish papules on the right side of her forehead. The number of lesions had gradually increased, developing into erythematous infiltrated plaques and nodules with scales, and then extending to the upper right side of the face and almost the whole forehead. The lesions were persistent over 5 years, and the patient had no previous intervening medical treatment prior to referral to the authors' outpatient department for evaluation of the skin lesions. The patient did not recall any potential inducement or any possibility of receiving immunosuppressive therapy. Since the onset of the disease, the patient had not complained of systemic discomfort. Her general condition was good, and she did not have a history of hepatitis or tuberculosis.

Dermatological examination revealed a large area of erythematous infiltrated plaques and nodules with scales, sporadic ulcers, and scars on the upper right side of the patient's face and almost her whole forehead (Figure 1). No cervical or axillary lymph nodes were palpable.

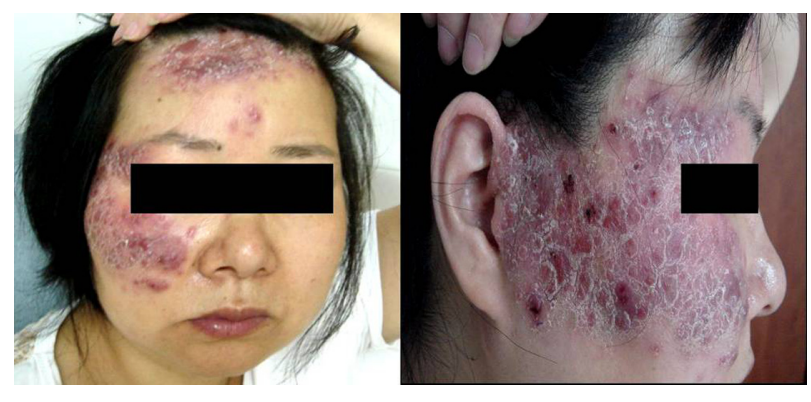

Figure I Skin lesions presented as a large area of erythematous infiltrated plaques and nodules with scales, sporadic ulcers, and scabs on the upper right side of the patient's face and almost the entire forehead.

No active pulmonary disease was detected by chest X-ray. Routine laboratory tests showed no noteworthy abnormalities. The results of human immunodeficiency virus antibody detection (conducted twice over 3 months) were negative. Cell-mediated immunity levels in peripheral blood cells from the patient were detected using a flow cytometer, (BD FACSCalibur ${ }^{\mathrm{TM}} ; \mathrm{BD}$ Bioscience, San Jose, CA) and the CD4-positive T-cell count in the peripheral blood cells, as well as other cell counts, was normal. Specific stains (such as periodic acid-Schiff and Giemsa stains) for microorganisms were negative. Ziehl-Neelsen staining results were positive. Tissue cultures showed that colonies grown in LöwensteinJensen (L-J) medium at two different temperatures $\left(32^{\circ} \mathrm{C}\right.$ and $37^{\circ} \mathrm{C}$ ) for $2-3$ weeks developed pigmentation in the dark and in the light. Fungal and other standard bacterial cultures were negative. A skin biopsy, taken from a lesion on the patient's forehead, stained with hematoxylin and eosin showed hyperplastic epidermis and epithelioid cell granulomas that were composed of tissue cells and multinucleated giant cells; infiltration with lymphocytes and eosinophils was further observed (Figure 2).

During diagnostic evaluation, the patient was empirically administered oral clarithromycin and moxifloxacin. At the

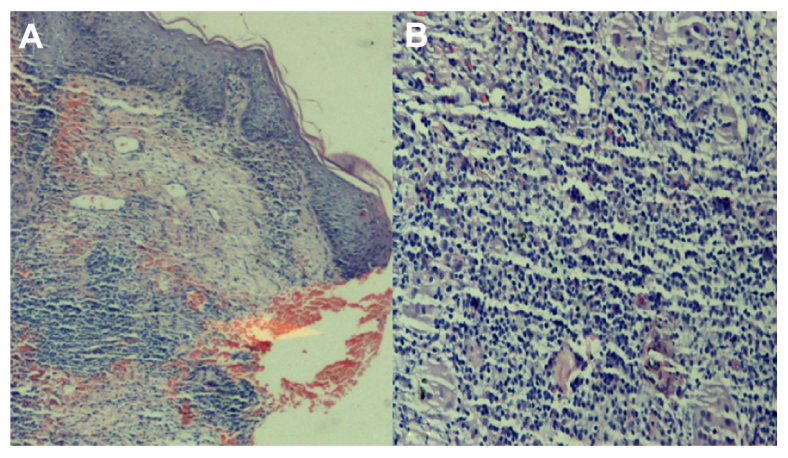

Figure 2 Histopathology with hematoxylin and eosin staining showed infective granuloma and multinucleated giant cells: (A) infective granuloma in dermis (original magnification, $\times 40$ ); (B) epithelioid cells and multinucleated giant cells (original magnification, $\times 200)$. 
first stage of treatment, the lesions noticeably improved, and half of them were cured. However, during administration of the drugs, the platelet count declined to $30 \times 10^{9} / \mathrm{L}$, which was considered to be an adverse reaction of moxifloxacin. After withdrawal from moxifloxacin, the palate count increased but the condition of the lesions deteriorated and the lesions became enlarged. Clarithromycin, amikacin, and ethambutol were then administered, resulting in slow improvement in the patient.

\section{Methods}

\section{Identification of mycobacterial isolate by acid-fast staining, drug susceptibility testing, and conventional procedures}

A lesioned skin tissue homogenate smear was stained by the Ziehl-Neelsen method. Samples of lesioned skin were inoculated on L-J medium and separately incubated at two different temperatures $\left(32^{\circ} \mathrm{C}\right.$ and $\left.37^{\circ} \mathrm{C}\right)$ in the dark and light, respectively. A test for the possible production of pigmentation was conducted on the isolated strain. Ziehl-Neelsen staining was used to confirm whether or not the cultured organisms were acid-fast bacilli (AFB). The samples were also inoculated on different media to detect bacteria and fungus. In vitro drug susceptibility testing was performed on the isolated strain using the microtiter plate method in accordance with Clinical and Laboratory Standards Institute guidelines. ${ }^{9}$

\section{Preparation of template DNA}

One loop of bacteria was harvested from the L-J medium slope incubated at $37^{\circ} \mathrm{C}$ and suspended in $2 \mathrm{~mL}$ of sterile distilled water. Samples were then frozen in liquid nitrogen and transferred to boiling water five times to release mycobacterial DNA. After centrifugation to pellet insoluble debris, the supernatant was used as the DNA template for PCR.

\section{PCR}

Two oligonucleotide primers (forward, 5'-ACCAACGATGGTGTGTCCAT-3'; reverse, 5'-CTTGTCGAACCGCATACCCT-3') were used to amplify a 439 bp fragment of the mycobacterial hsp65 gene. Two other oligonucleotide primers (forward, 5'-GAGATACTCGAGTGGCGAAC-3'; reverse, 5'-GGCCGGCTACCCGTGGTC-3') were used to amplify a $208 \mathrm{bp}$ fragment of the mycobacterial $16 \mathrm{~S}$ rDNA sequence. ${ }^{10}$ PCR was performed in a $50 \mu \mathrm{L}$ reaction volume containing $10 \mu \mathrm{L}$ of the DNA template, $1.25 \mathrm{U}$ of Taq polymerase (Promega Corporation, Madison, WI), a $10 \times$ reaction buffer, $200 \mu \mathrm{mol} / \mathrm{L}$ of deoxyribonucleoside triphosphate, $2.5 \mathrm{mmol} / \mathrm{L}$ of magnesium chloride, and $1 \mu \mathrm{mol} / \mathrm{L}$ of each primer. The thermal profile for the amplification of the mycobacterial hsp65 gene involved initial denaturation at $94^{\circ} \mathrm{C}$ for 5 minutes; 45 cycles of $94^{\circ} \mathrm{C}$ for 1 minute, $60^{\circ} \mathrm{C}$ for 1 minute, and $72^{\circ} \mathrm{C}$ for 1 minute; and then a 10 -minute extension at $72^{\circ} \mathrm{C}$. The PCR conditions for the amplification of the mycobacterial $16 \mathrm{~S} r D N A$ sequence involved incubation for 10 minutes at $40^{\circ} \mathrm{C}$ and 40 cycles of $94^{\circ} \mathrm{C}$ for 1.5 minutes, $65^{\circ} \mathrm{C}$ for 2 minutes, and $72^{\circ} \mathrm{C}$ for 3 minutes. After the last PCR cycle, the vials were maintained at $72^{\circ} \mathrm{C}$ for 10 minutes. The presence of amplified products was confirmed by agarose gel electrophoresis. ${ }^{11-13}$

\section{PCR-RFLP and gene sequencing for hsp65 and I6S rDNA genes}

The amplification products $(10 \mu \mathrm{L})$ were separately incubated with BstEII (5 U) and HaeIII (5 U) endonucleases (MBI Fermentas Inc, Burlington, ON, Canada) at $37^{\circ} \mathrm{C}$ for 3 hours. The digestion products $(10 \mu \mathrm{L})$ were analyzed by electrophoresis on 2\% MetaPhor Agarose gel (FMC BioProducts, Rockland, MD) and stained with ethidium bromide. A photograph of the restriction patterns was taken, and this photo was visually analyzed to determine the number of fragments present. The sizes of the fragments were compared with the molecular size standard using a 100 bp marker (GeneRuler 100 bp DNA Ladder; MBI Fermentas Inc). Restriction fragments shorter than $60 \mathrm{bp}$ were not considered because they were presumed to be primer or primer dimer bands. The data were analyzed based on a report by Ena et al. ${ }^{14}$ The PCR products of $h s p 65$ and $16 \mathrm{~S} r D N A$ genes were sequenced, and the BLAST (Basic Local Alignment Search Tool) program was used to compare the sequences of the isolated strain with those of other mycobacterial species in the GenBank database (http://www. ncbi.nlm.nih.gov/BLAST) and the PRAsite database (http:// app.chuv.ch/prasite/index.html).

\section{Results}

The lesioned skin tissue homogenate smear with ZiehlNeelsen staining was positive. Slow growth of the smooth colonies was noted after $2-3$ weeks of incubation at $32^{\circ} \mathrm{C}$ and $37^{\circ} \mathrm{C}$. The pigmentation production test revealed a yellowpigmented mycobacterial species that was scotochromogenic, belonging to Runyon's group II. Ziehl-Neelsen staining confirmed that the cultured organisms were AFB (Figure 3). Fungal and other standard bacterial cultures were negative.

In vitro susceptibility testing using the absolute concentration method in accordance with Clinical and Laboratory Standards Institute guidelines showed that 


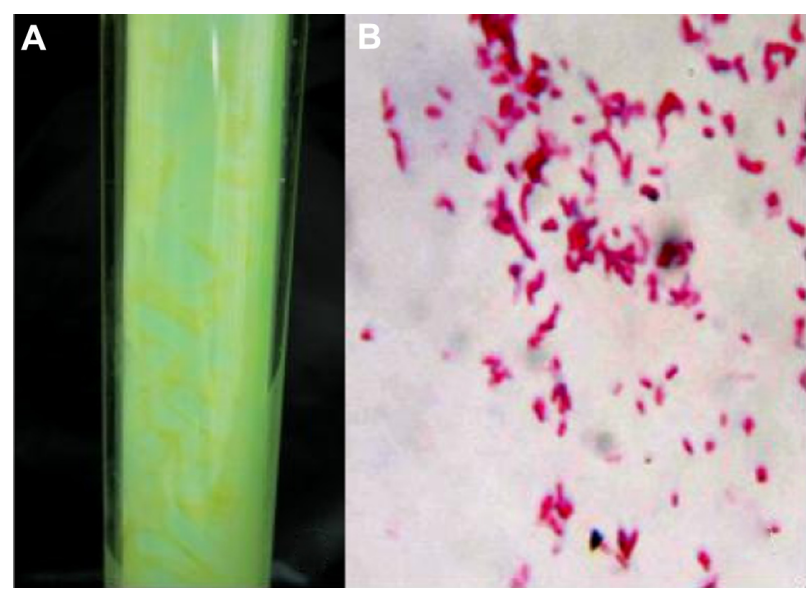

Figure 3 Cultured organisms incubated at $37^{\circ} \mathrm{C}$ in the dark for about 20 days: (A) smooth colonies with a yellow pigment formed; (B) Ziehl-Neelsen staining revealed the organisms were acid-fast bacilli.

the isolated organism was sensitive to clarithromycin and moxifloxacin and was moderately sensitive to amikacin.

A fragment of $439 \mathrm{bp}$, encoding mycobacterial $65 \mathrm{kDa}$ heat shock protein, was amplified in the strain isolated from the patient. Digestion of the PCR product yielded two fragments of 235/120/85 bp with BstEII and three fragments of 130/9 bp with HaeIII (Figure 4). The RFLP pattern of the isolated AFB was identical to that of $M$. parascrofulaceum.

Sequencing of the hsp65 and $16 S$ rDNA genes showed $100 \%$ and $99.9 \%$ similarity, respectively, with M. parascrofulaceum.

\section{Discussion}

M. parascrofulaceum, a new species of NTM, is classified as group II by the Runyon classification because of its

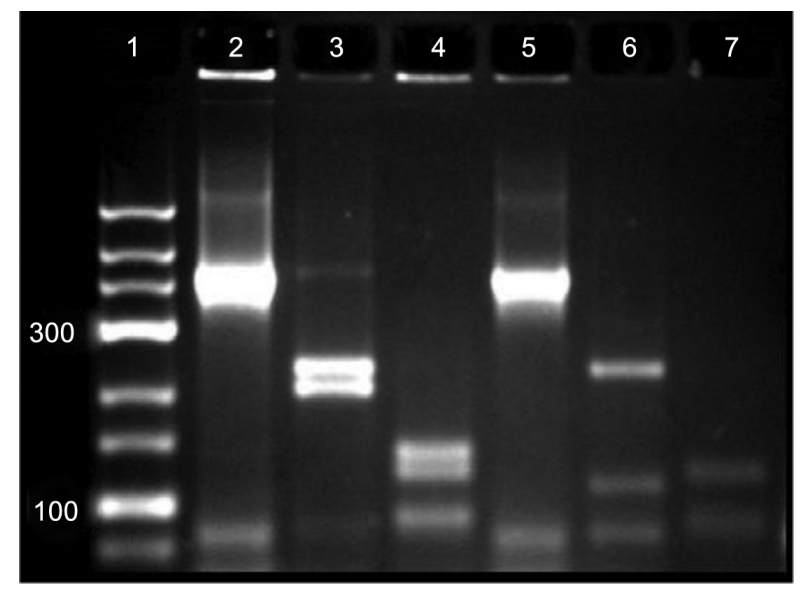

Figure 4 Restriction analysis of isolated strain and Mycobacteria scrofulaceum amplification products: lane I, DNA size marker (from upper to lower: 700, 500, $400,300,200,150,100,75$, and 50 bp); lanes 2-4 (for M. scrofulaceum), undigested amplification products, BstEll digests, and Haelll digests; lanes 5-7 (for the isolated strain), undigested amplification products, BstEll digests, and Haelll digests. slow growth and scotochromogenicity. The morphology and biochemical profile of $M$. parascrofulaceum are indistinguishable from those of Mycobacterium scrofulaceum; genotypically, it is most closely related to Mycobacterium simiae. M. parascrofulaceum can grow on L-J medium at $25^{\circ} \mathrm{C}-37^{\circ} \mathrm{C}$ (optimally at $37^{\circ} \mathrm{C}$ ), with tolerance to $5 \%$ sodium chloride. ${ }^{4}$ It can also survive and persist in extreme habitats under highly acidic conditions and high temperatures (up to $56^{\circ} \mathrm{C}$ ). Thus, this strain can be found in the acidic hot springs of Yellowstone National Park. ${ }^{15}$ While no report yet confirms so, the strain is believed to exist in natural and municipal water and soil.

Similar to NTM, M. parascrofulaceum is an opportunistic pathogen that can cause infections in the lungs, bronchi, lymph nodes, and genitals, especially in both older patients with underlying diseases and immunosuppressed individuals with AIDS; its preferred target appears to be the lung. However, cases of $M$. parascrofulaceum are infrequently reported. Since Turenne et al's ${ }^{4}$ identification of the species in 2004, only seven cases of M. parascrofulaceum infection have been reported (Table 1), and almost all these cases showed underlying diseases (four had AIDS and four had respiratory diseases or an epidermoid carcinoma). One case involved high-risk sexual behavior but showed no evidence of immunodeficiency. Four cases involved the lung; three other infective targets were the bronchus, genitals, or blood (bacteremia). The symptoms observed in these cases were atypical, involving only fever, cough, and pain; some cases were even asymptomatic. ${ }^{4-8}$ To the best of the authors' knowledge, no case of cutaneous infection with $M$. parascrofulaceum has been reported before now. To the best of the authors' knowledge, this paper is the first to report on a case in which the skin was the only infected organ involved, manifesting with asymptomatic reddish papules, nodules, plaques, and patches on the right side of the patient's face that persisted for 5 years. No evidence of immunosuppression or base disease was found. Testing for the human immunodeficiency virus antibody was negative, and the CD4-positive T-cell count was normal. No active pulmonary disease was found by chest radiography. ZiehlNeelsen stains and tissue cultures were positive. A skin biopsy taken from the lesion on the patient's forehead showed infective granulomas. The diagnosis of NTM infection was not difficult, but exclusion of several suspicious diagnoses such as fungal or bacterial infections, cutaneous tuberculosis, leprosy, and inflammatory or tumor disease was necessary. As treatment and prognosis depend on the recognition of species, identification of the pathogen was important but 


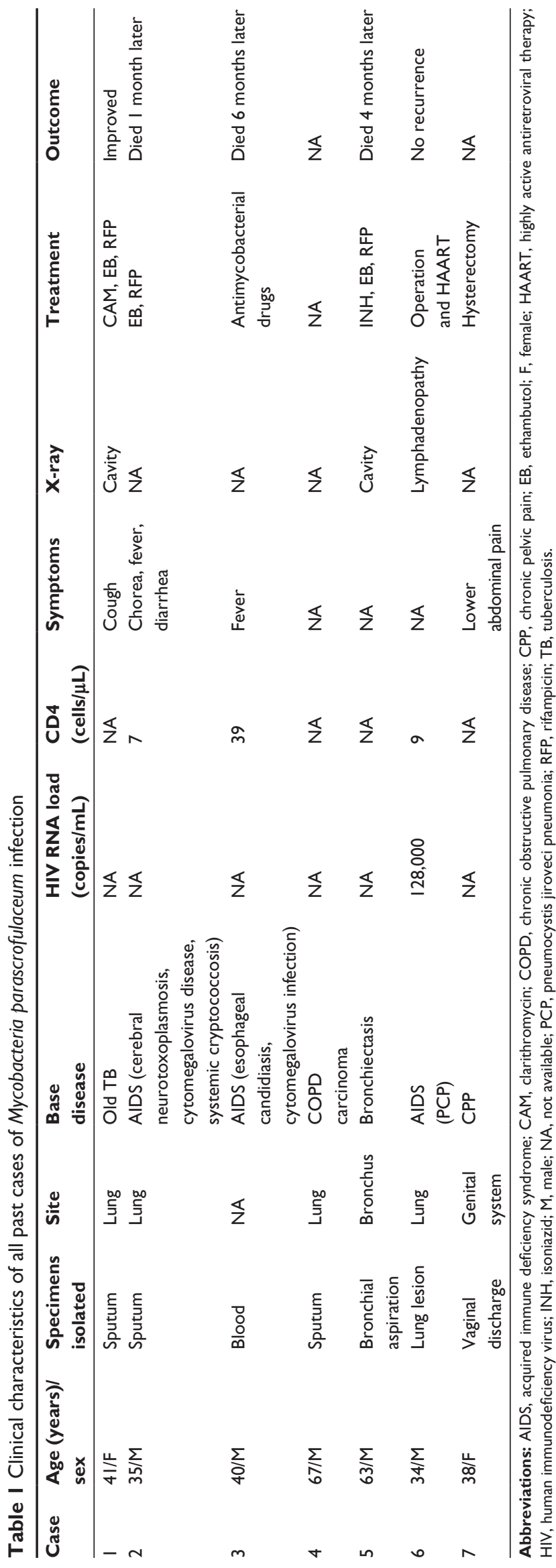

complex. Except for conventional examinations such as in vitro culture, Ziehl-Neelsen staining, and drug susceptibility testing, further novel laboratory tests such as PCR, PCRRFLP analysis, genetic sequencing analysis, commercial DNA probing, high-performance liquid chromatography, and the SNaPshot ${ }^{\circledR}$ technique (Applied Biosystems, Foster City, CA) were necessary to identify NTM at the species level. In the present case, PCR-RFLP and gene sequencing for $h s p 65$ and $16 S$ rDNA showed that the organism was $M$. parascrofulaceum.

The new organism that Turenne et $\mathrm{al}^{4}$ found in 2004 was susceptible to rifampicin, clarithromycin, amikacin, and sulfamethoxazole but resistant to isoniazid in in vitro drug susceptibility tests. Later studies suggested that the strain was also sensitive to levofloxacin. According to several reports, rifampicin, clarithromycin, and new quinolones are key drugs for the treatment of such an organism. ${ }^{4,6,7}$ The present study shows that the isolated organism is sensitive to clarithromycin, rifampicin, moxifloxacin, and amikacin, in accordance with previous studies. In the present case, treatment with clarithromycin and moxifloxacin was effective. Moxifloxacin was withdrawn because of the adverse reactions. The curative effects of amikacin and ethambutol were not as significant as those of clarithromycin and moxifloxacin.

The prognosis of $M$. parascrofulaceum infection remains unclear. Although antimycobacterial drugs were used including the susceptible ones, most of the reported cases have resulted in death. In two known cases in which the patient survived, one case improved by clarithromycin, ethambutol, and rifampicin treatment and the other did not show recurrence after a surgical debridement of the pathological lesions in lung and lymph node without any chemotherapy. The prognosis of M. parascrofulaceum infection may depend on the immunity of patients, especially those with AIDS. ${ }^{4-8}$

\section{Conclusion}

The diagnosis of NTM cutaneous infection of unremarkable appearance requires a dermatologist with a high index of suspicion to perform adequate and precise examinations in order to identify the relevant species and to help rule out tuberculosis. Once diagnosed, the conditions of the NTMinfected patient should be evaluated. Empirical treatments may be feasible. Drug susceptibility tests may be necessary to address different responses to therapies between species.

Cases of M. parascrofulaceum infection are rare. Little information on M. parascrofulaceum infection or therapies for treating this organism is available. In the case presented, the authors isolated the AFB from lesioned skin and identified 
them as $M$. parascrofulaceum using the biochemical method. The authors' diagnosis and their experience with the treatment of cutaneous infection by $M$. parascrofulaceum were discussed here to improve the current knowledge of infections caused by this bacterium.

\section{Acknowledgments}

This study was supported by grants from the National Natural Science Foundation of China (No 30972651) and the fund for the Key Clinical Program of the Ministry of Health of the People's Republic of China (No 2010-2012-125). The authors thank all of the medical workers in the hospital ward for their cooperation and clinical assistance and acknowledge all of the workers in the hospital's mycobacteria laboratory for their technical guidance.

\section{Disclosure}

The authors report no conflicts of interest in this work. The authors alone are responsible for the content and writing of this paper.

\section{References}

1. Tortoli E. The new mycobacteria: an update. FEMS Immunol Med Microbiol. 2006;48(2):159-178.

2. Cook JL. Nontuberculous mycobacteria: opportunistic environmental pathogens for predisposed hosts. Br Med Bull. 2010;96:45-59.

3. Taylor TB, Patterson C, Hale Y, Safranek WW. Routine use of PCRrestriction fragment length polymorphism analysis for identification of mycobacteria growing in liquid media. J Clin Microbiol. 1997; 35(1):79-85.

4. Turenne CY, Cook VJ, Burdz TV, et al. Mycobacterium parascrofulaceum sp. nov, novel slowly growing, scotochromogenic clinical isolates related to Mycobacterium simiae. Int J Syst Evol Microbiol. 2004;54(Pt 5): 1543-1551.
5. Tortoli E, Chianura L, Fabbro L, et al. Infections due to the newly described species Mycobacterium parascrofulaceum. J Clin Microbiol. 2005;43(8):4286-4287.

6. Teruya H, Tateyama M, Hibiya K, et al. Pulmonary Mycobacterium parascrofulaceum infection as an immune reconstitution inflammatory syndrome in an AIDS patient. Intern Med. 2010;49(16):1817-1821.

7. Shojaei H, Hashemi A, Heidarieh P, Daei-Naser A. Chronic pelvic pain due to Mycobacterium parascrofulaceum in an Iranian patient: first report of isolation and molecular characterization from Asia. Braz J Infect Dis. 2011;15(2):186-187.

8. Hibiya K, Tateyama M, Teruya H, et al. Immunopathological characteristics of immune reconstitution inflammatory syndrome caused by Mycobacterium parascrofulaceum infection in a patient with AIDS. Pathol Res Pract. 2011;207(4):262-270.

9. National Committee for Clinical Laboratory Standards (NCCLS). Susceptibility Testing of Mycobacteria, Nocardiae, and Other Aerobic Actinomycetes: Approved Standard. NCCLS document M24-A. Wayne, PA: NCCLS; 2003.

10. Kox LF, van Leeuwen J, Knijper S, Jansen HM, Kolk AH. PCR assay based on DNA coding for $16 S r R N A$ for detection and identification of mycobacteria in clinical samples. J Clin Microbiol. 1995;33(12): 3225-3233.

11. Wang H, Jin P, Wu Q. Disseminated cutaneous infection with Mycobacterium abscessus in a patient with a low CD4+ T cell count. Eur J Dermatol. 2008;18(3):337-340.

12. Feng Y, Xu H, Wang H, Zhang C, Zong W, Wu Q. Outbreak of a cutaneous Mycobacterium marinum infection in Jiangsu Haian, China. Diagn Microbiol Infect Dis. 2011;71(3):267-272.

13. Wang H, Wu Q, Lin L, Cui P. Cutaneous tuberculosis: a diagnostic and therapeutic study of 20 cases. J Dermatolog Treat. 2011;22(6): 310-314.

14. Ena P, Sechi LA, Saccabusi S, et al. Rapid identification of cutaneous infections by nontubercular mycobacteria by polymerase chain reactionrestriction analysis length polymorphism of the hsp65 gene. Int J Dermatol. 2001;40(8):495-499.

15. Santos R, Fernandes J, Fernandes N, Oliveira F, Cadete M. Mycobacterium parascrofulaceum in acidic hot springs in Yellowstone National Park. Appl Environ Microbiol. 2007;73(15):5071-5073.
Therapeutics and Clinical Risk Management

\section{Publish your work in this journal}

Therapeutics and Clinical Risk Management is an international, peerreviewed journal of clinical therapeutics and risk management, focusing on concise rapid reporting of clinical studies in all therapeutic areas, outcomes, safety, and programs for the effective, safe, and sustained use of medicines. This journal is indexed on PubMed Central, CAS,

\section{Dovepress}

EMBase, Scopus and the Elsevier Bibliographic databases. The manuscript management system is completely online and includes a very quick and fair peer-review system, which is all easy to use. Visit http://www.dovepress.com/testimonials.php to read real quotes from published authors. 